\title{
Neuromodulation Improves the Evolution of Forward Models
}

\author{
Mohammad Sadegh Norouzzadeh \\ Evolving Al Lab \\ Computer Science Dept. \\ University of Wyoming \\ mnorouzz@uwyo.edu
}

\author{
Jeff Clune \\ Evolving Al Lab \\ Computer Science Dept. \\ University of Wyoming \\ jclune@uwyo.edu
}

\begin{abstract}
Many animals predict the outcomes of their actions by internal models. Such "forward models" enable animals to rapidly simulate many actions without performing them to choose an appropriate action. Robots would similarly benefit from forward models. However, such models must change over time to account for changes in the environment or body, such as injury. Thus, forward models must not only be accurate, but also adaptable. Neural networks can learn complex functions with high accuracy, hence they are suitable candidates to build forward models for robots. Generally, neural networks are static, which means once they pass the training phase, their weights remain unchanged and they thus cannot adapt themselves if something about the world or their body changes. Plastic neural networks change their connections over time via local learning rules (e.g. Hebbian rule) and can thus deal with unforeseen changes. A more complex, yet still biologically-inspired, technique is neuromodulation, which can change per-connection learning rates in different contexts. In this paper, we test the hypothesis that neuromodulation may improve the evolution of forward models because it can heighten learning after drastic changes such as injury. We compare forward models evolved with neuromodulation to those evolved with static neural networks and Hebbian plastic neural networks. The results show that neuromodulation produces forward models that can adapt to changes significantly better than the controls. Our findings suggest that neuromodulation is an effective tool for enabling robots (and artificial intelligence agents, more generally) to have more adaptable, effective forward models.
\end{abstract}

\section{Keywords}

Forward Models, Plastic Neural Networks, Neuromodulation

Permission to make digital or hard copies of all or part of this work for personal or classroom use is granted without fee provided that copies are not made or distributed for profit or commercial advantage and that copies bear this notice and the full citation on the first page. Copyrights for components of this work owned by others than the author(s) must be honored. Abstracting with credit is permitted. To copy otherwise, or republish, to post on servers or to redistribute to lists, requires prior specific permission and/or a fee. Request permissions from permissions@ acm.org.

GECCO '16, July 20 - 24, 2016, Denver, CO, USA

(C) 2016 Copyright held by the owner/author(s). Publication rights licensed to ACM. ISBN 978-1-4503-4206-3/16/07 . \$15.00

DOI: http://dx.doi.org/10.1145/2908812.2908837

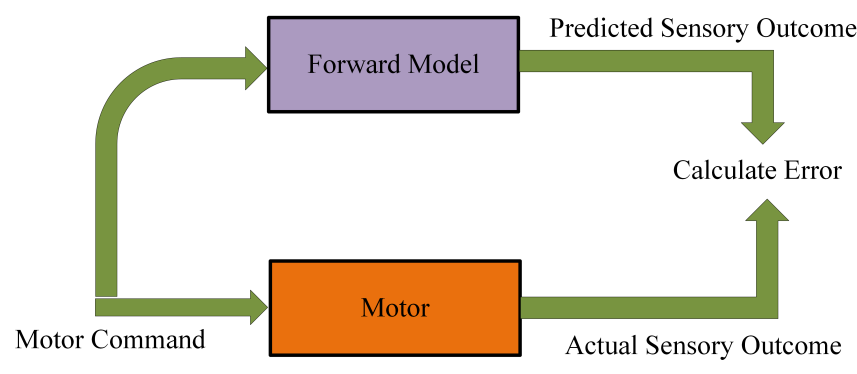

Figure 1: The overall diagram of a typical forward model. Forward models receive a copy of motor commands and predict the sensory outcomes of such movements. The differences between the predictions and actual sensory outcomes (i.e. errors) are supplied to improve future predictions.

\section{INTRODUCTION AND BACKGROUND}

Living animals such as insects, birds, and humans employ internal models that allow them to predict the sensory outcomes of their movements [31,33]. These models take an internal copy of an action-generating signal and predict the sensory outcomes that will result from that action. A famous experiment that showed the existence of forward models in animals is the Helmholtz experiment [33]. Helmholtz was a 19th-century German physician and physicist who suggested that the brain predicts the position of the eye based on the movement signal rather than actually sensing the eye's position. He found that when the eye is moved by an external source the brain wrongly feels that the world around it is moving [33]. His experiment shows that there is an internal model in the brain that predicts the position of the eye according to the movement signal. Another fact supporting the existence of forward models is that we cannot tickle ourselves because our brains predict what happens when we stimulate ourselves, but other people can tickle us because our brain does not have copies of their movement signals, hence it cannot predict the outcome [2].

Robots can similarly benefit from internal models because such models could help robots predict the sensory outcomes of their movements. Being able to predict the consequences of actions could grant robots the ability to better adapt to changes to their environments or bodies; specifically, they could mentally simulate possible movements to select an appropriate one without having to incur the costs and hazards of trying each action the real world $[3,16]$. Such models can 


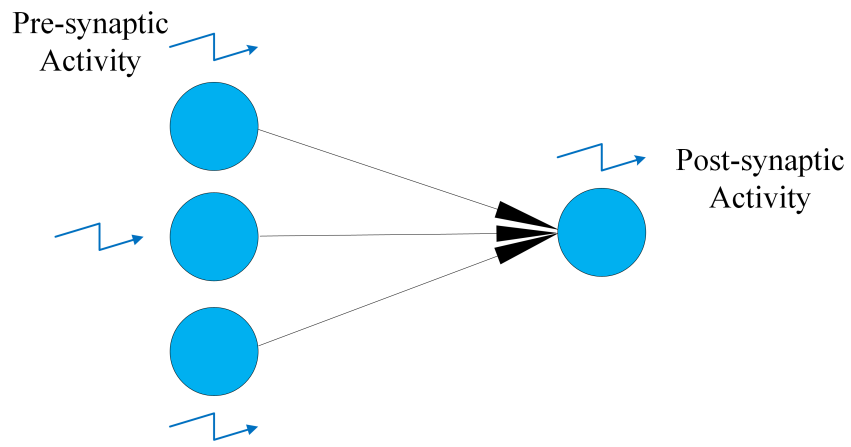

(a) Hebbian Plasticity

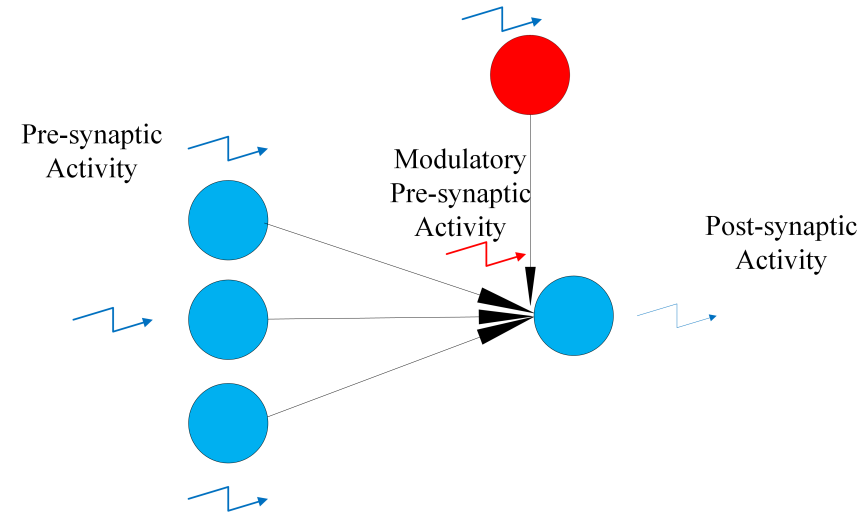

(b) Neuromodulation

Figure 2: Neuromodulation enables fine-grained control over local learning rules. (a) In plain Hebbian learning, a connection strength is updated as a function of pre- and post-synaptic activity only. (b) With neuromodulation, the rate of learning can be up-regulated or down-regulated in different contexts depending on the data. That regulation occurs via a modulatory signal output by modulatory neurons within the network (red circle). Thus, parts of a neural network recognize contexts in which the learning rates of individual connections in the network should be increased and decreased. For example, learning could be turned off if a model is error-free, but turned on if the model begins producing errors (e.g. after fatigue or damage occurs).

also enable robots to model other agents, whether humans or other robots, improving their ability to plan and respond accordingly [4].

Formally, a forward model is a function that at each time step $t$ takes a motor command $C^{t}$ as input and predicts the sensory outcomes $S^{t}$ as output (Fig. 1). After the predictions have been made, we can calculate the difference between the predictions and actual sensory outcomes (i.e. error) and those error signals can be used to enhance the predictions of the forward model. Many modern robots take advantage of forward models, but there are two major issues with current forward models.

The first is that they are often manually designed by humans for a specific environment. As robots and the tasks they perform grow more complex, this approach becomes increasingly untenable. Accordingly, researchers have developed several methods for automatically building forward models. Dearden and Demiris [7] proposed an adaptable autonomous forward model based on Bayesian networks and applied it to predict outcomes of motor commands. In their experiments, they had an arm with two motors along with a simple vision system to capture the outcomes. Moriguchi and Lipson [16] proposed a framework for learning forward models via symbolic regression; they applied their model for motion planning and control of a motored pendulum.

The second drawback to most current forward models is that they are static (i.e. unchanging). If the environment does change, either the forward models fail completely or they must be retrained from scratch. An example of the latter is Bongard, et al. [3]. Instead of creating a single, adaptable forward model, they efficiently synthesize a set of (non-adaptable) models and actively improve that set to find a new model that is accurate given the drastic change that has occurred.

This paper makes two major contributions. The first is to introduce a method that evolves adaptable (i.e. plastic) forward models. The second is to test whether neuromodu- lation improves the evolution of such forward models. The results confirm that evolution can automatically make effective forward models and the performance of such models is improved when they are evolved with neuromodulatory learning.

\subsection{Neural Networks}

In animals, the nervous system is responsible for building internal models. Artificial Neural Networks or simply Neural Networks (NN) are a class of computational models inspired by animal brains that consist of several interconnected computational elements (neurons). Each neuron takes signals from its inputs, processes them, and passes the result through weighted connections to interconnected neurons. Training a neural network involves adjusting its weights to compute a useful output as a function of its inputs. After we train a neural network it can predict outputs for novel inputs. Neural networks are able to model complex input-output relationships, making them suitable for building internal models. Here, we automatically design neural networks that serve as forward models for a simple robot.

Because we are interested in learning not only how to produce effective forward models for robotic control, but also to learn about the evolution of forward models in animals, the forward models in this paper are produced by evolving neural networks, a field called neuroevolution $[5,6,10$, $12,27]$. Neuroevolution (NE) trains neural networks with evolutionary algorithms (EAs), some of which can evolve both the structure (i.e. topology) and weights of neural networks given a measure of performance [5, 10,27]. Specifically, NE builds a population of candidate networks and then refine them by applying small changes (mutations) [10]. These small changes may include adding or removing neurons, adding or removing a random connection, changing the weight of a connection, changing the type of a neuron (standard or modulatory), and so on.

$\mathrm{NE}$ methods can work in reinforcement learning situations 
in which the correct output is not known for every input shown to the network during training. Instead, NE only requires a measure of performance to be periodically provided (e.g. at the end of a trial that includes exposing a network to many unlabeled inputs). For example, in robot navigation, it is easy to evaluate the final position of the robot to see if it is near a goal, but it is difficult to provide a set of correct actions for each situation the robot encounters. NE has been applied successfully for a variety of problems including Atari game playing [14], control [13,30], and automatic feature selection [32].

\subsection{Plasticity}

Neural networks usually have two phases. The first is a training phase and the second is a testing phase in which the trained network predicts outputs for unseen inputs. Usually, NNs are static, which means we only change their weights during their training phase, after which the weights remain unchanged during testing while the NN makes decisions.

An ideal forward model would be able to respond to unforeseen changes that occur within the lifetime of the organism. Generally, if a change occurs in the environment, static NNs cannot adapt appropriately because their weights are unchangeable. On the other hand, plastic neural networks are capable of adjusting their structure (structural plasticity) or weights (synaptic plasticity) during their lifetime [28], making them capable of behavioral change and providing more behavioral robustness [29].

Synaptic plasticity, which we limit our focus to in this paper, can come via local learning rules that change weights $[1,11,21]$. In general, each connection within a network could have its own learning rule. The general form of a local learning rule is given in Eq. 1 and 2, where $w_{n e w}$ is the new weight of the connection, $w_{\text {old }}$ is the old weight of the connection, $\Delta w$ is the change of weight, $\Phi$ is the learning rule, $o_{\text {pre }}$ is pre-synaptic activity, and $o_{\text {post }}$ is post-synaptic activity.

$$
\begin{gathered}
w_{\text {new }}=w_{\text {old }}+\Delta w \\
\Delta w=\Phi\left(o_{\text {pre }}, o_{\text {post }}, w_{\text {old }}\right)
\end{gathered}
$$

A well-known local learning rule formula is the plain Hebbian rule (Eq. 3), which is applied to all of the connections of a network [15]. The plain Hebbian rule can be generalized to specific types of pre- or post-synaptic correlations [25], but here we focus on the simpler, more commonly used version in Eq. 3. During training and testing phases, an input is presented to the network, its output for that input is calculated, pre- and post-synaptic activities are captured, a learning rate $(\eta)$ is applied, and each weight is updated. This cycle then repeats for the next input.

$$
\Delta w=\eta \cdot o_{\text {pre }} \cdot o_{\text {post }}
$$

\subsection{Neuromodulation}

In the brain, some neurons control the learning behavior of the connections between other neurons by releasing one or more chemical transmitters. This phenomenon is usually referred to as neuromodulation [24]. Inspired by this natural phenomenon, researchers have added an abstraction of neuromodulation to NNs to improve short-term memory [34], evolvability [23], learning and adaptability [26], and finegrained plasticity control [24].
Following nature, in the NN abstraction of neuromodulation, some neurons called modulatory neurons control the plasticity of connections between other neurons (Fig. 2). That requires two types of neurons: standard neurons and modulatory neurons [24]. Each neuron $i$ has two ways it can be activated: standard activations $a_{i}$ come from standard neurons (Eq. 4), and modularity activations $m_{i}$ come from modularity neurons (Eq. 5). In Eq. 4 and Eq. 5, $i$ and $j$ are neuron indices, $w_{j i}$ is the connection weight between neuron $j$ and neuron $i$, and $o_{j}$ is the post-synaptic activity of neuron $j$.

$$
\begin{aligned}
a_{i} & =\sum_{j \in S t d} w_{j i} \cdot o_{j} \\
m_{i} & =\sum_{j \in M o d} w_{j i} \cdot o_{j}
\end{aligned}
$$

With neuromodulation, weight changes are computed by Eq. 6. In comparison with other plasticity models, neuromodulation enables fine-grained control over plasticity because it grants the capability of turning local rules on or off for some neurons dynamically depending on the data.

$$
\Delta w_{j i}=\tanh \left(m_{i} / 2\right) \cdot \eta \cdot\left[o_{i} \cdot o_{j}\right]
$$

\section{METHODS}

In this paper, in order to automatically build forward models, we evolve neural networks. To build adaptable forward models (those that can change during an organism's lifetime), we evolve plastic neural networks. Specifically, we compare two different types of learning: simple Hebbian learning and neuromodulation. Our test problem is to build a forward model for a simple robot arm (Fig. 3) that experiences changes over time (specifically, fatigue and damage). This paper tests the hypothesis that neuromodulation helps to produce more adaptable forward models. For this purpose, we compare a static NN model, a Hebbian plastic NN model, and a neuromodulation-controlled Hebbian plastic NN model.

\subsection{Test Problem}

The test problem involves a robot arm moved by a single degree of freedom at its base that can rotate 360 degrees. The robot is exposed to several sequences of commands within its lifetime. Initially, the arm is set to 0 degrees (due East). Applying each motor command sets a desired angle for the arm. For example, with a motor command of 90 degrees, the arm moves to 90 degrees (due North). After applying each command, we reset the position of the arm to the original position ( 0 degrees). The goal of the forward model is to predict the coordinates ( $x$ and $y$ ) of the arm's end effector (i.e. the endpoint of the arm) given the motor command that is applied to the joint. The mathematical formula for the position of the end effector in the absence of any changes is shown in Eq. 7 and 8, where $\theta$ indicates the motor command (desired angle) and $l$ indicates the length of the arm, which is 1 in our experiments.

$$
\begin{aligned}
& x=l \cdot \cos (\theta) \\
& y=l \cdot \sin (\theta)
\end{aligned}
$$




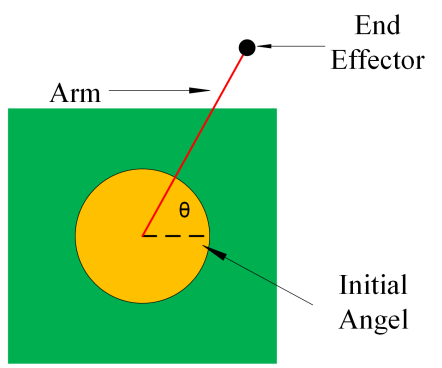

(a) Normal Conditions

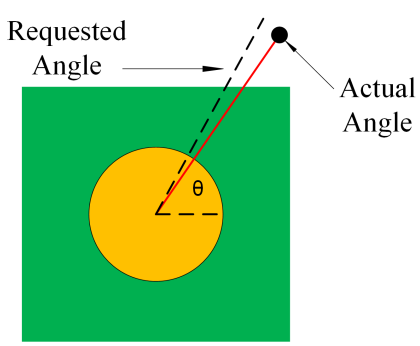

(b) Fatigue

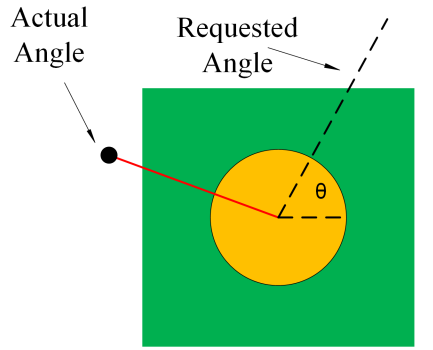

(c) Damage

Figure 3: The simple robot arm from our experiments. (a) In normal conditions, a motor command of $\theta$ will cause the arm to move to that angle. (b) In case of fatigue, a motor command of $\theta$ will cause the arm to move to slightly less than $\theta$. (c) In case of damage, a motor command of $\theta$ does not have any effect because the arm is stuck at a previous angle.

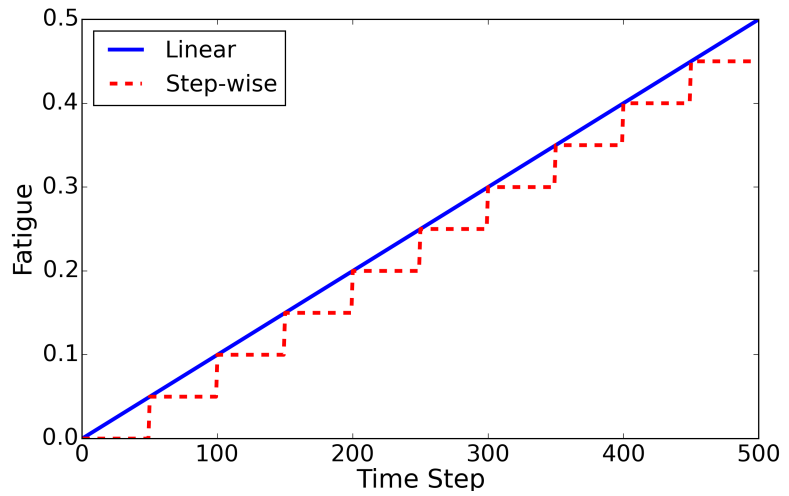

Figure 4: Linear and step-wise fatigue variants. With linear fatigue, fatigue increases linearly over time. With step-wise fatigue, fatigueincreases in several discrete steps.

We test two different types of changes: fatigue and damage. For each sequence of motor commands, these two phenomena may happen with an independent probability of 0.5 . Thus, there are four possible conditions: neither fatigue nor damage (i.e. normal conditions), fatigue only, damage only, and both fatigue and damage. The details of how fatigue and damage are implemented are explained next.

\subsubsection{Fatigue}

In idealized situations (e.g. simulation), when we send a motor command to the joint of a robot, it performs that command without error. Muscle fatigue is a well-known phenomenon for humans; when our muscles are very tired, we lose some of our control over them [22]. Robot motors may similarly suffer from fatigue because of extreme motor temperatures or batteries dropping in voltage [20]. Here we simulate fatigue for robot motors by setting motors to a fraction of the motion that would have occurred due to the command in normal conditions. For example, in the presence of fatigue, if we give a command of 30 degrees to the joint, the arm does not move all the way from its initial position of 0 degrees to the desired angle, meaning the final angle will be less than 30 degrees. Fatigue increases over time, meaning that the fraction of the requested angle that is achieved decreases over time (Eq. 9).

$$
\hat{\theta}=(1-\text { fatigue }) \cdot \theta
$$

In Eq. $9, \theta$ is a motor command and $\hat{\theta}$ is the actual angle that the arm moves to. Here we test with two different types of fatigue: linear fatigue and step-wise fatigue (Fig. 4). With linear fatigue, we increase the amount of fatigue linearly to $50 \%$ by the end of the motor command sequence (Eq. 10). In step-wise fatigue we increase the fatigue in a fixed number of discrete steps (here 10) to $45 \%$ (Eq. 11). In Eq. 10 and 11, $N$ indicates the length of the command sequences and $i$ is the index of a motor command in the sequence.

$$
\begin{gathered}
\hat{\theta}_{i}=\theta_{i} *\left(1-\frac{i}{2 N}\right) \\
\hat{\theta}_{i}=\theta_{i} *\left(\left\lfloor\frac{10 i}{N}\right\rfloor * 0.05\right)
\end{gathered}
$$

\subsubsection{Damage}

The second type of drastic change a robot may have to cope with is extreme damage to a motor (e.g. the motor stops working entirely). We simulate that by making damage randomly occur at some time in the second half of the motor sequence, after which the robot's joint is not able to follow any command and remains fixed at the angle it was set to when the damage occurred. Because our simple robot has only one motor, when damage occurs the position of the end effector that the forward model is trying to predict remains fixed. These dynamics are described in the following equation:

$$
\hat{\theta}_{i}=\left\{\begin{array}{cc}
\theta_{i} & i<t \\
\theta_{t-1} & i>=t
\end{array}\right.
$$

where $i$ is the index of a motor command in the sequence and $t$ is the instant that the damage occurs. Damage overwrites fatigue, therefore, if we have both damage and fatigue, we ignore the fatigue and just keep the last angle.

\subsection{Algorithm Details}

Forward models must be able to detect if their predictions are incorrect in order to improve. If we only input the motor command to the forward model, there will be no way for 


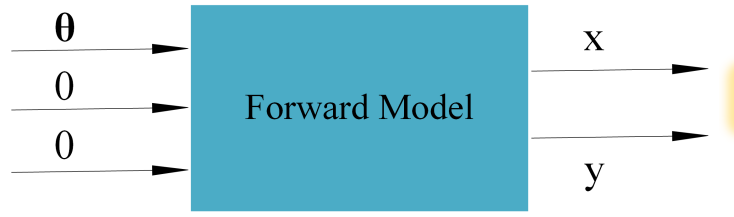

(a) Step 1

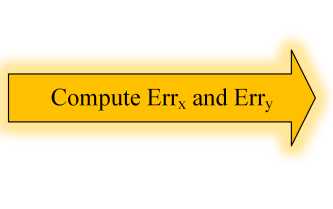

(b) Step 2

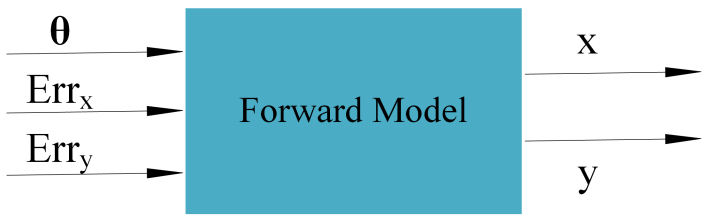

(c) Step 3

Figure 5: The three steps of the command implementation process. (a) The first neural network pass: a motor command and zeros are input into the model and the neural network forward model is run to compute predictions. (b) Error signals are computed from the first pass, which determine fitness. Note that this step does not require an update of the neural network. (c) The second neural network pass: the motor command and error signals from the first pass are fed into the model so that its learning algorithm can adjust the weights of the model. The error signals from this pass are ignored and do not affect fitness.

the forward model to notice changes. In order to enable the forward model to improve itself if it makes errors, we also input the differences between the predicted coordinates of the end effector and the actual coordinates (i.e. error signals) into the network (Eq. 13 and 14).

$$
\begin{gathered}
\text { error }_{x}=\left|x_{\text {actual }}-x\right| \\
\text { error }_{y}=\left|y_{\text {actual }}-y\right| \\
\text { error }=\text { error }_{x}+\text { error }_{y}
\end{gathered}
$$

Therefore, our forward models have 3 inputs (a motor command, the error signal of $x$, and the error signal of $y$ ) and 2 outputs (predicted $x$ coordinate and $y$ coordinate of the end effector). In order to provide the error signals for the network, we have three steps for inputting each motor command into the network (Fig. 5). In the first step, we input the motor command into the network (for this step, the error inputs are set to zero) and compute the predictions. In the second step, we calculate the prediction error signals. In the third step, we input both the motor command and the error signals again. The fitness function only considers the predictions of the first step. The purpose of the third step is to provide the network the magnitude of its prediction errors so its learning algorithm can modify the weights of the model to improve future predictions. Any prediction errors that result in this third step do not affect fitness.

We evolve networks with the NeuroEvolution of Augmenting Topologies (NEAT) algorithm [27] because it is widely used and because it can evolve both the structure and the weights of networks. Because NEAT has been described in detail in numerous previous papers, we only describe it briefly here, for details about it, see [27]. NEAT starts with a population of simple two-layer NNs that become more complex over time via mutations that add nodes and connections. Solutions are also refined by mutations that change connection weights. Like all evolutionary algorithms, NEAT needs a criterion for comparing the performance of various candidate solutions (i.e. a fitness function), which is described in the next section.

\subsection{Fitness Evaluation}

The fitness for a network is a score summarizing its performance over its entire lifetime. During its lifetime, each net- work is exposed to 10 sequences of motor commands. Each sequence is composed of 500 motor commands. Each motor command is an angle the motor is commanded to move to. Each individual motor command involves a three-step command implementation process (outlined in Fig. 5) that involves running the $\mathrm{NN}$ twice (the first pass provides the command and no error signals, and the second provides the command again along with the error signals from the first pass). The fitness function only incorporates errors produced after the first pass (Fig. 5).

Each sequence is randomly allocated to one of four conditions: (1) no fatigue-no damage, (2) fatigue-only, (3) damageonly, (4) fatigue and damage. Thus, every network has a chance of encountering any of these conditions and must handle it appropriately. The existence of different conditions, including a condition without damage or fatigue, prevents the network from adopting simple open-loop hacks to solve the problem, such as approximately solving fatigue via a constant decay schedule.

Thus, in total, each network is run twice per command, for 10 sequences of 500 motor commands each, for a total of $2 \times 500 \times 10=10,000$ network updates per lifetime. Because only the error of the first pass affects fitness, the error vector is of length $1 \times 500 \times 10=5000$ for both $x$ and $y$ outputs, producing a total error vector of length 10,000 per fitness evaluation. Those 10,000 numbers are summed to produce the total error, and fitness is its negative (Eq. 16).

$$
\text { fitness }=-\sum_{1}^{\text {No.ofSeq. }} \sum_{1}^{N} \text { error }
$$

Adding behavioral diversity is known to be beneficial for finding better solutions to deceptive problems $[17,19]$. We thus use a multi-objective evolutionary algorithm with two objectives: increasing fitness and increasing behavioral diversity of the population. Specifically, experiments are performed with the multi-objective Non-dominated Sorting Genetic Algorithm II (NSGA-II) [8] as implemented in the Sferes framework [18]. The parameters for running the algorithm are presented in Table 1.

We measure behavioral diversity as the average of behavioral distance between each pair of individuals in the population. Specifically, for each individual, we store all of the outputs of the first pass of the network for both $x$ and $y$ for all motor commands from all sequences (thus, 10,000 outputs in total). We then compute the Euclidean distance 
Table 1: The evolutionary algorithm parameters.

\begin{tabular}{|c|c|}
\hline Parameter & Value \\
\hline \hline Add Connection Rate & 0.2 \\
\hline Add Neuron Rate & 0.2 \\
\hline Remove Neuron Rate & 0.2 \\
\hline Remove Connection Rate & 0.2 \\
\hline Change Type of a Neuron Rate & 0.2 \\
\hline Change Connection Weight Rate & 0.2 \\
\hline $\begin{array}{c}\text { Change Activation Function Rate } \\
\text { (Sigmoid, Tanh, Linear, Sin., Cos.) }\end{array}$ & 0.2 \\
\hline Probability of a Neuron Being Modulatory & 0.3 \\
\hline Hebbian Learning Rate & 0.05 \\
\hline Minimum Value of a Connection weight & -5 \\
\hline Maximum Value of a Connection weight & +5 \\
\hline Minimum Number of Neurons & 5 \\
\hline Maximum Number of Neurons & 20 \\
\hline Minimum Number of Connections & 3 \\
\hline Maximum Number of Connections & 100 \\
\hline
\end{tabular}

between these arrays to obtain pairwise distances between individuals. Each individual's diversity is then its mean distance to every other member of the population.

\section{EXPERIMENTS AND RESULTS}

In order to evaluate the effectiveness of the neuromodulation technique for building adaptable forward models, we compare three models: the static NN forward model, the plain Hebbian plastic NN forward model, and the neuromodulation-based forward model. We design two set of experiments: one in which fatigue is linear, and another in which fatigue progresses in a step-wise manner (Fig. 4). Each experiment is run 35 times.

Fig. 6 shows NN error values for the best model from each treatment on an example motor command sequence from each of the four different possible conditions: (1) normal (no fatigue or damage), (2) fatigue-only, (3) damage-only, and (4) fatigue-and-damage conditions. The static model is very accurate at normal sequences and damage-only sequences before the damage occurs, but it cannot adapt to the damage-induced change because it is unable to change its weights during its lifetime. The strategy evolution seems to adopt, if there is no error, is to predict as if there were no fatigue or damage for all sequences. When there is an error, it adjusts its output based on that error, but cannot utilize that information to reduce error anywhere near zero. The plain Hebbian forward model can change its weights during its lifetime, but it does not evolve an intelligent way to make changes at appropriate times (e.g. only after damage) to make extremely accurate predictions. In stark contrast, the neuromodulation-controlled forward model has low errors in throughout all four conditions, except for a few updates after extreme damage (spikes in the error plots) as it rapidly adapts its model to account for the sudden change (Fig. 6). While less visually apparent, the lack of error in the fatigue treatments also demonstrates that neuromodulation is slowly and accurately adapting its forward model as fatigue builds.

The quantitative results averaged over all runs support these qualitative observations. In both sets of experiments (with linear and step-wise fatigue), forward models evolved with neuromodulation significantly outperform the models evolved in both the Hebbian and static treatments across evolutionary time (Fig. $7, p<0.05)$. That trend holds when comparing final populations $\left(p<10^{-13}\right)$.

Overall, models evolved with plain Hebbian learning do not perform substantially better than static models. In the set of experiments with linear fatigue, there is no significant difference between the two treatments after initial generations (Fig. 7, $p>0.05$ ). Surprisingly, in the set of experiments with step-wise fatigue, the static model actually significantly outperforms the Hebbian model, although the difference is small (Fig. $7, p<0.05$ ). These results underscore the importance of neuromodulation when evolving plastic neural network controllers. In situations when plasticity is required, Hebbian plasticity alone may not be a powerful enough form of plasticity. Instead, to truly see the benefits of plasticity, evolution must be given the power to regulate learning in different situations based on the data.

\section{CONCLUSION}

In many applications, robots need to deal with various unforeseen changes in their body and their environment. Predicting all possible changes in order to handle all possible circumstances is infeasible. Therefore, modern robots need accurate, adaptable forward models to adapt themselves to various changes, such as changes to the environment or their bodies like damage. Such models enable robots to simulate dangerous actions without performing them to select an appropriate action.

This paper introduced a method for evolving adaptable forward models and tested the hypothesis that neuromodulation improves the evolution of forward models. The data show that neuromodulation produces significantly better forward models than Hebbian learning and also significantly outperforms static models. The paper also demonstrates that Hebbian learning is not able to provide the required plasticity required for adaptable forward models, at least in our experiments.

Overall, the performance of the neuromodulation forward models is very impressive. Error is near zero in a wide range of conditions, from when there is neither fatigue nor damage to when there are both. As expected and required, error spikes just after a major change, but the model quickly adapts to account for it. That said, our experiments are in the very simple setup of a robot arm controlled by one motor. It will be an interesting question for future research to what extent neuromodulation can enable the evolution of accurate, adaptable forward models for more complex robots.

Additionally, it has previously been shown that neuromodulation can enable organisms to adapt to changing environments $[9,21,24,25]$. That work and our new results suggest that neuromodulation could enable the evolution of forward models that model environmental changes as well as changes to an agent's body. Such models should ultimately improve the ability of robotics to plan ahead and select appropriate actions without costly, real-world trial and error.

\section{ACKNOWLEDGMENTS:}

We thank all of the members of the Evolving AI Lab for their feedback and support, especially Lucas Helms, Henok Mengistu, Anh Nguyen, and Joost Huizinga. JC was supported by an NSF CAREER award (CAREER: 1453549). 


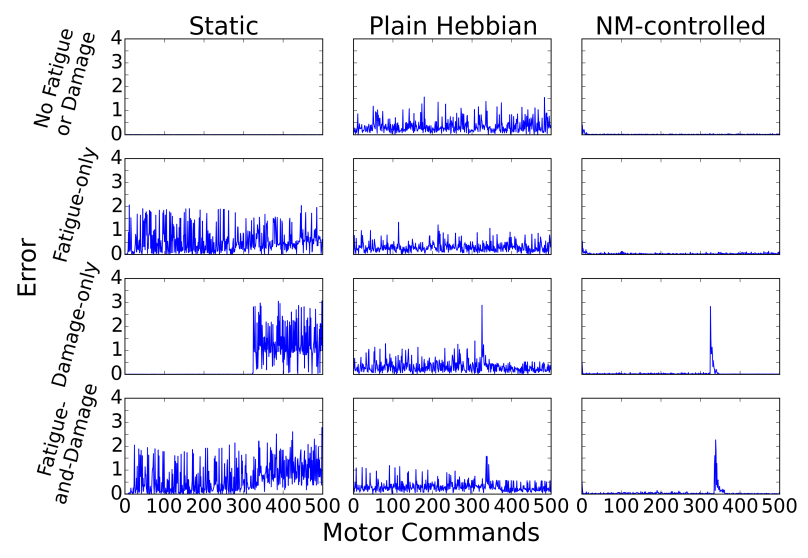

(a) Linear Fatigue

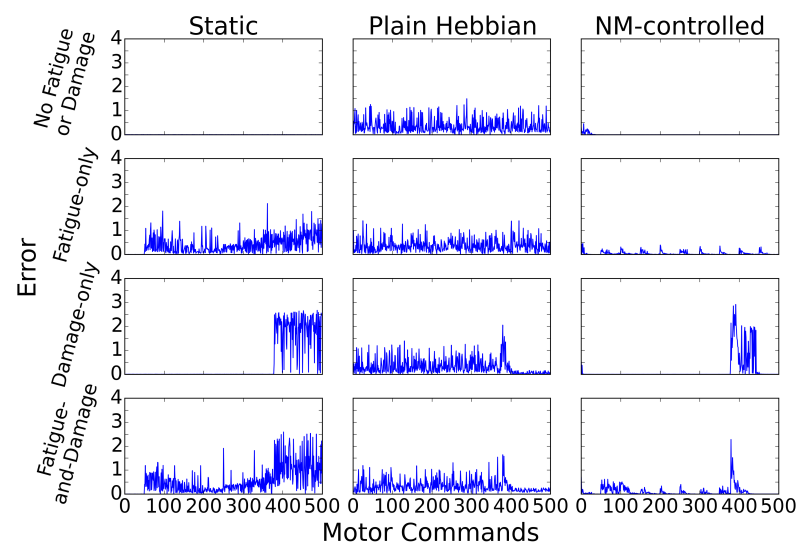

(b) Step-wise Fatigue

Figure 6: Error of the best model from each treatment on example sequences from each damage condition. The static model performs well under normal conditions, but it fails to adapt to changes. The plain Hebbian is not able to effectively adapt to reduce error. In stark contrast, forward models evolved with neuromodulation exhibit close to the theoretical minimum error: they have almost no error throughout, except right after major changes. They quickly adapt the model to account for such changes, reducing error back to near zero.

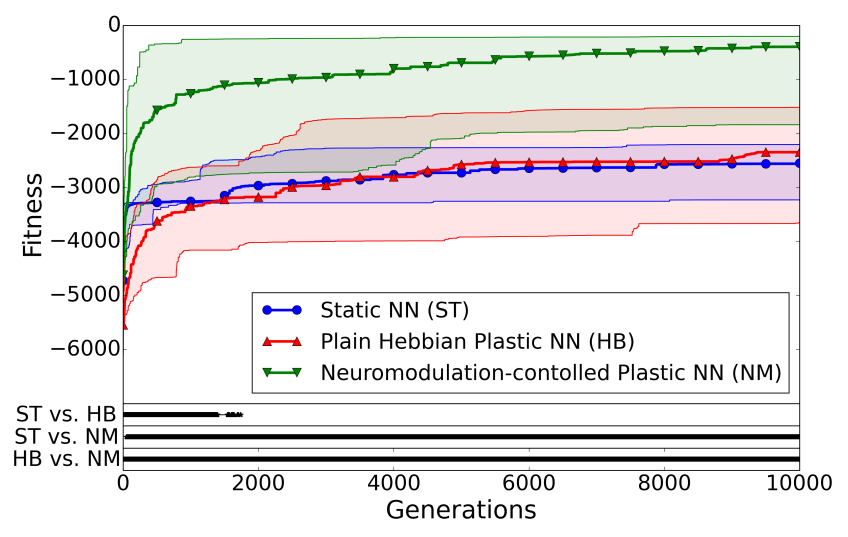

(a) Linear Fatigue

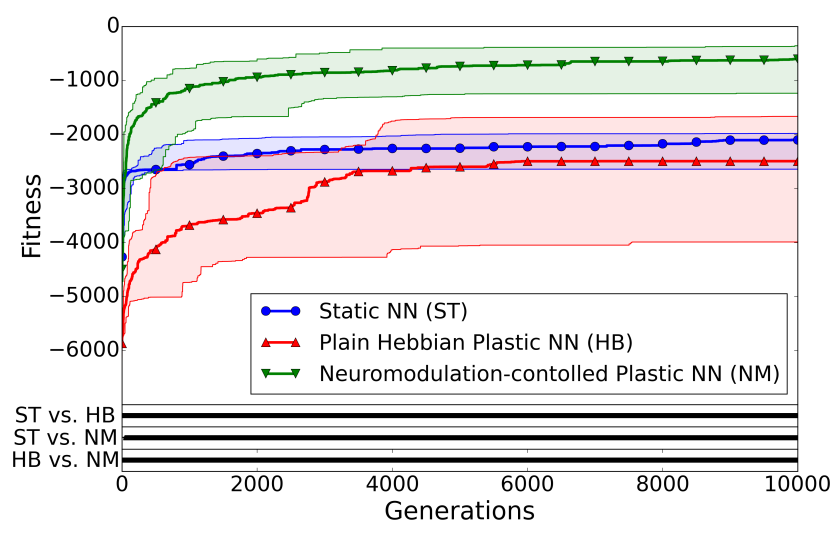

(b) Step-wise Fatigue

Figure 7: Forward models evolved with neuromodulation are significantly better at predicting the outcomes of their actions and adapting to changes such as damage and fatigue. In experiments with linear fatigue (a) and stepwise fatigue (b), the error produced by neuromodulation models is significantly lower across evolutionary time than that produced with static models or Hebbian learning. Below the plot, an asterisk is shown if a significant difference exists between each pair of treatments at a threshold of $p<0.05$. Often, $p$ values are far lower than this threshold. For example, comparing mean performance in the final generation, the $p$ value is $p<10^{-13}$ comparing neuromodulation to the other treatments. The performance differences between static models and Hebbian models is slight. In the linear fatigue case (a) it is not significant after initial generations. In the stepwise fatigue case (b), the static model actually significantly outperforms the Hebbian model. The overall conclusion is that, even when plasticity is required, Hebbian plasticity is not powerful enough. To truly realize the benefits of plasticity, a more powerful form of plasticity like neuromodulation is required. Plotted are the median and range of fitness values (minimum and maximum) across 35 independent runs for the static, plain Hebbian, and neuromodulation forward models. All $p$ values here and elsewhere in the paper are computed by a Mann-Whitney U test.

\section{REFERENCES}

[1] Jonathan Baxter. The evolution of learning algorithms for artificial neural networks. Complex systems, pages 313-326, 1993.

[2] Sarah-J Blakemore, Daniel M Wolpert, and Chris D
Frith. Central cancellation of self-produced tickle sensation. Nature neuroscience, 1(7):635-640, 1998.

[3] Josh Bongard, Victor Zykov, and Hod Lipson. Resilient machines through continuous self-modeling. Science, 314(5802):1118-1121, 2006.

[4] Cynthia Breazeal and Brian Scassellati. Robots that 
imitate humans. Trends in cognitive sciences, 6(11):481-487, 2002.

[5] Jeff Clune, Benjamin E. Beckmann, Charles Ofria, and Robert T. Pennock. Evolving coordinated quadruped gaits with the hyperneat generative encoding. In Proceedings of the IEEE Congress on Evolutionary Computation, pages 2764-2771, 2009.

[6] Jeff Clune, Kenneth O. Stanley, Robert T. Pennock, and Charles Ofria. On the performance of indirect encoding across the continuum of regularity. IEEE Transactions on Evolutionary Computation, 15(4):346-367, 2011.

[7] Anthony Dearden and Yiannis Demiris. Learning forward models for robots. In IJCAI, volume 5, page 1440, 2005.

[8] Kalyanmoy Deb, Amrit Pratap, Sameer Agarwal, and TAMT Meyarivan. A fast and elitist multiobjective genetic algorithm: Nsga-ii. Evolutionary Computation, IEEE Transactions on, 6(2):182-197, 2002.

[9] Kai Olav Ellefsen, Jean-Baptiste Mouret, and Jeff Clune. Neural modularity helps organisms evolve to learn new skills without forgetting old skills. PLoS Comput Biol, 11(4):e1004128, 2015.

[10] Dario Floreano and Claudio Mattiussi. Bio-inspired artificial intelligence: theories, methods, and technologies. The MIT Press, 2008.

[11] Dario Floreano and Joseba Urzelai. Evolutionary robots with on-line self-organization and behavioral fitness. Neural Networks, 13(4):431-443, 2000.

[12] Jason Gauci and Kenneth O. Stanley. Autonomous evolution of topographic regularities in artificial neural networks. Neural Computation, 22(7):1860-1898, 2010.

[13] Faustino John Gomez and Risto Miikkulainen. Robust non-linear control through neuroevolution. Computer Science Department, University of Texas at Austin, 2003.

[14] Matthew Hausknecht, Joel Lehman, Risto Miikkulainen, and Peter Stone. A neuroevolution approach to general atari game playing. Computational Intelligence and AI in Games, IEEE Transactions on, 6(4):355-366, 2014.

[15] Donald Olding Hebb. The organization of behavior: A neuropsychological theory. Psychology Press, 2005.

[16] Hirotaka Moriguchi and Hod Lipson. Learning symbolic forward models for robotic motion planning and control. In Proceedings of European Conference of Artificial Life (ECAL 2011), pages 558-564, 2011.

[17] Jean-Baptiste Mouret and Stéphane Doncieux. Overcoming the bootstrap problem in evolutionary robotics using behavioral diversity. In Evolutionary Computation, 2009. CEC'09. IEEE Congress on, pages 1161-1168. IEEE, 2009.

[18] Jean-Baptiste Mouret and Stéphane Doncieux. SFERESv2: Evolvin' in the multi-core world. In Proc. of Congress on Evolutionary Computation (CEC), pages 4079-4086, 2010.

[19] Jean-Baptiste Mouret and Stéphane Doncieux. Encouraging behavioral diversity in evolutionary robotics: An empirical study. Evolutionary computation, 20(1):91-133, 2012.

[20] Veljko Potkonjak, Jelena Radojicic, Spyros Tzafestas, and Dragan Kostic. Modeling robot "psycho-physical" state and reactions-a new option in human-robot communication part 2: Modeling and simulation. Journal of Intelligent and Robotic Systems, 35(4):353-364, 2002.

[21] Sebastian Risi and Kenneth O Stanley. Indirectly encoding neural plasticity as a pattern of local rules. In From Animals to Animats 11, pages 533-543. Springer, 2010.

[22] Kent Sahlin. Muscle fatigue and lactic acid accumulation. Acta physiologica Scandinavica. Supplementum, 556:83-91, 1985.

[23] Tom Smith, Phil Husbands, Andy Philippides, and Michael O'Shea. Neuronal plasticity and temporal adaptivity: Gasnet robot control networks. Adaptive Behavior, 10(3-4):161-183, 2002.

[24] Andrea Soltoggio, John A Bullinaria, Claudio Mattiussi, Peter Dürr, and Dario Floreano. Evolutionary advantages of neuromodulated plasticity in dynamic, reward-based scenarios. In ALIFE, pages 569-576, 2008.

[25] Andrea Soltoggio, P Durr, Claudio Mattiussi, and Dario Floreano. Evolving neuromodulatory topologies for reinforcement learning-like problems. In Evolutionary Computation, 200\%. CEC 200\%. IEEE Congress on, pages 2471-2478. IEEE, 2007.

[26] Olaf Sporns and William H Alexander. Neuromodulation and plasticity in an autonomous robot. Neural Networks, 15(4):761-774, 2002.

[27] Kenneth O Stanley and Risto Miikkulainen. Evolving neural networks through augmenting topologies. Evolutionary computation, 10(2):99-127, 2002.

[28] Thomas Trappenberg. Fundamentals of computational neuroscience. Oxford University Press, 2010.

[29] Joseba Urzelai and Dario Floreano. Evolution of adaptive synapses: Robots with fast adaptive behavior in new environments. Evolutionary Computation, 9(4):495-524, 2001.

[30] Vinod K Valsalam, Jonathan Hiller, Robert MacCurdy, Hod Lipson, and Risto Miikkulainen. Constructing controllers for physical multilegged robots using the enso neuroevolution approach. Evolutionary Intelligence, 5(1):45-56, 2012.

[31] Barbara Webb. Neural mechanisms for prediction: do insects have forward models? Trends in neurosciences, 27(5):278-282, 2004.

[32] Shimon Whiteson, Peter Stone, Kenneth O Stanley, Risto Miikkulainen, and Nate Kohl. Automatic feature selection in neuroevolution. In Proceedings of the 2005 conference on Genetic and evolutionary computation, pages 1225-1232. ACM, 2005.

[33] Daniel M Wolpert and J Randall Flanagan. Motor prediction. Current Biology, 11(18):R729-R732, 2001.

[34] Tom Ziemke and Mikael Thieme. Neuromodulation of reactive sensorimotor mappings as a short-term memory mechanism in delayed response tasks. Adaptive Behavior, 10(3-4):185-199, 2002. 